\title{
Mosquito hearing is the most sensitive among arthropods - but is the sound level of a male swarm loud enough to be picked up by the female's particle-velocity sensor?
}

\author{
L. Feugère ${ }^{1,2}$, G. Gibson ${ }^{2}$, O. Roux ${ }^{1,3}$ \\ ${ }^{1}$ MIVEGEC, IRD, CNRS, Univ. Montpellier, Montpellier, France. \\ ${ }^{2}$ Natural Resources Institute, University of Greenwich, Chatham, Kent ME4 4TB, UK \\ ${ }^{3}$ Institut de Recherche en Sciences de la Santé (IRSS), 01 BP 545 Bobo-Dioulasso 01, Burkina Faso
}

Males of many mosquito species aggregate in station-keeping swarms, waiting for the arrival of conspecific females to mate with. We test whether audition could be used by a female to locate male swarms and to assess whether the males are conspecific. The sound level resulting from thousands of wing flaps could be loud enough to be heard at long range $(\sim 1 \mathrm{~m})$ via the antennal flagellum (particle velocity sensor, primarily designed for close-range communication).

A mosquito hears a conspecific by adjusting its own wing-beat frequency so that the difference tone between its own and the opposite-sex frequencies falls into a narrow band to which the auditory organ is tuned. Indeed, the antennal flagella produce distortion products resulting in difference tones of the nearby soundscape.

Swarms of males were recorded and played-back to females in a $2 \mathrm{~m}$-sided flight chamber. The natural sounds of the males of two species (Anopheles coluzzii and An. gambiae) and related synthetic sounds were played at different sound levels to individual free-flying An. coluzzii females. The mosquitoes' responses were investigated by analysing changes in 3D-tracked flight trajectories and wing-beat frequencies. The results show: 1) females do respond to the sound of swarming males, 2) a qualitative difference between female and male behaviour, 3 ) a quantitative effect of the sound stimulus of conspecific males, and 4) verification of previous results suggesting the importance of the first harmonic of their wing beats in mosquito acoustic communication. 